\title{
Frequency ratios and the discrimination of pure tone sequences
}

\author{
E. GLENN SCHELLENBERG \\ University of Windsor, Windsor, Ontario, Canada \\ and \\ SANDRA E. TREHUB \\ University of Toronto, Mississauga, Ontario, Canada
}

\begin{abstract}
We examined the effect of frequency ratios on the discrimination of patterns of alternating pure tones (ABABA). Listeners heard a repeating pattern presented in transposition (same frequency ratios between successive tones, different absolute frequencies) and were required to indicate when the pattern changed (different frequency ratios and absolute frequencies). Changes from patterns with simple frequency ratios to those with more complex ratios were more readily detected than were changes from complex ratios to simpler ratios.
\end{abstract}

Musical tunes that differ in their starting tone are perceived as equivalent if the relations among the successive tones are preserved (Attneave \& Olson, 1971). This proclivity to process pitch relationally may include processing advantages for some relations between tones over others (Schellenberg \& Trehub, 1994; Trehub \& Trainor, 1993). Thus, auditory patterns structured in particular ways may function as prototypes, being more readily perceived and represented than other patterns (Trehub \& Unyk, 1991). Presumably, all musical systems reflect such processing predispositions.

The focus of the present study was on the simplest possible relations between successive tones, notably intervals (i.e., relations between two tones). One consequence of inherent processing advantages for some intervals over others would be privileged occurrence of such intervals cross-culturally. Other consequences would be more rapid learning, superior retention, and possibly a preference for musical pieces incorporating such intervals. It follows, moreover, that enhanced processing of specific intervals should be independent of formal musical training.

One possible basis for musical prototypes is the simplicity of frequency ratios between tones (Jones, 1990), defined by the integers in the ratio: the smaller the integers, the simpler the ratio. The perceptual similarity of

This research, which was supported by the Natural Sciences and Engineering Research Council of Canada, was submitted by E.G.S. in partial fulfillment of the requirements of the $\mathrm{PhD}$ at Cornell University. We thank Richard E. Pastore and Michael P. Lynch for comments on an earlier version of the manuscript. Correspondence should be addressed to E. G. Schellenberg, Department of Psychology, University of Windsor, Windsor, ON, Canada N9B 3P4, or to S. E. Trehub, Centre for Research in Human Development, University of Toronto, Erindale Campus, Mississauga, ON, Canada L5L 1 C6. tones an octave apart (frequency ratio of $2: 1$ ) is universal (Dowling \& Harwood, 1986; Lerdahl \& Jackendoff, 1983), being apparent in infants as young as 3 months of age (Demany \& Armand, 1984). Octaves are unique, however, in that tones an octave apart have the same tone chroma (i.e., the distinction between any tone called $\mathrm{X}$ and any tone called $\mathrm{Y}$ ) as well as the simplest frequency ratio of any interval (other than a unison). Thus, the perceptual equivalence of tones separated by an octave may result either from their simple frequency ratio or from their identical tone chroma. Cross-cultural examination of scale structure reveals a preponderance of perfect fifths $(3: 2)$ and perfect fourths $(4: 3)$ as well as octaves (see Sachs, 1943), which is consistent with the notion that simple frequency ratios are inherently easy to process.

Indeed, the simplicity of frequency ratios has played a central role in theories of intervallic consonance and dissonance. Sensory consonance is often distinguished from musical consonance. The former refers to consonance based on physical (i.e., acoustic) factors, and is, therefore, independent of musical conventions. Sensory consonance, which is considered to be a function of critical bandwidth (Kameoka \& Kuriyagawa, 1969; Plomp \& Levelt, 1965), refers to the absence of amplitude fluctuations in two simultaneously sounded tones (because of their nonoverlapping critical bands); sensory dissonance refers to the "roughness" (very rapid amplitude fluctuations) that can result from simultaneously sounded tones with overlapping critical bands. By contrast, musical consonance is considered to result from tone compatibility, which is dependent on culture, convention, and context. Moreover, musical consonance is applicable to sequential as well as simultaneous tones. Consonant intervals occur between compatible tones and produce a feeling of stability, whereas dissonant intervals occur between incompatible tones and cause in- 
stability (e.g., Aldwell \& Schachter, 1989). Although the concepts of sensory and musical consonance differ, they are not completely independent (Bregman, 1990). For example, octaves have never been considered musically dissonant, and tones related by simple ratios, such as $3: 2$ and $4: 3$, are considered to be "stable" intervals across several musical cultures (Meyer, 1956).

Studies of successive pure tone intervals have accorded little attention to consonance, focusing instead on issues such as interval classification (Burns \& Ward, 1978; Siegel \& Siegel, 1977a, 1977b) and octave equivalence (Allen, 1967; Houtsma, 1968; Kallman, 1982; Thurlow \& Erchul, 1977). Nevertheless, the results provide little support for the claim that ratio simplicity is an important factor in perceptual processing. For example, evidence of octave equivalence has been absent in some instances (Kallman, 1982) and restricted to highly trained listeners in others (Allen, 1967; see also Thurlow \& Erchul, 1977), which is at odds with the evidence of octave equivalence in infancy (Demany \& Armand, 1984). Moreover, Burns and Ward (1978, Experiment 4) reported no evidence for natural categories of intervals based on the simplicity of frequency ratios.

A few studies of tone sequences have provided support for the simplicity of frequency ratios as a perceptual primitive. For example, adults are better at discriminating the temporal order of three-tone sequences when the tones are related by small-integer frequency ratios (Divenyi \& Hirsh, 1974). Even infants seem to exhibit enhanced processing of sequences with simple frequency ratios (Cohen, Thorpe, \& Trehub, 1987; Trainor \& Trehub, 1993a, 1993b; Trehub, Thorpe, \& Trainor, 1990). In none of the infant research, however, was simplicity of frequency ratios the specific focus of investigation.

The present study examined the possibility that some aspects of tone compatibility (i.e., musical consonance) in melody are based on acoustic properties of the stimulus and are independent of musical convention. Specifically, we inquired whether the simplicity of frequency ratios between successive pure tones affects ease of processing. If tones related by simple frequency ratios are fundamentally easier to process than those related by more complex ratios, then this processing advantage should be evident even in musically impoverished contexts with musically unsophisticated listeners.

Listeners were evaluated on their ability to discriminate intervals that differed by one semitone. For some groups of listeners, we evaluated the ability to detect changes in perfectly consonant intervals (i.e., tones related by very simple frequency ratios) that resulted in dissonant intervals (i.e., complex ratios). For other listeners, we evaluated the ability to detect changes in dissonant intervals that resulted in perfect consonances. We also examined more subtle changes that contrasted perfect and imperfect consonances (i.e., simple and moderately simple ratios). In line with music-theoretic conventions, ratios of $3: 2$ and $4: 3$ (perfect fifths and fourths) were classified as perfectly consonant, ratios of
5:4 and 8:5 (major thirds and minor sixths) as imperfectly consonant, and the ratio of $45: 32$ (tritone) as dissonant. The use of sequential pure tones (sine waves) sufficiently distant in pitch precluded traditional explanations of sensory consonance and dissonance as a function of overlapping critical bands, which could occur between simultaneous tones or between adjacent harmonics of successive complex tones. Moreover, the use of musically impoverished contexts (i.e., no musical key established) and listeners who were relatively unsophisticated musically minimized the effects of learned musical relations between tones.

The underlying assumption was that the accuracy of detecting changes in an auditory pattern would reflect its relative ease of processing. According to signal detection theory (Green \& Swets, 1974; Macmillan \& Creelman, 1991), superior performance results from a higher ratio of signal to noise. In the present experiment, the absolute size of the change to be detected (the signal), was held constant (one semitone) across conditions. What varied across conditions was the magnitude of background noise. A standard pattern with simple frequency ratios would minimize such noise by virtue of its hypothesized ease of processing and representation, leading to superior discrimination. Conversely, a standard pattern with complex frequency ratios would increase such noise by virtue of its hypothesized difficulty of processing and representation (i.e., a lower signal-tonoise ratio), resulting in poorer discrimination.

\section{METHOD}

\section{Subjects}

The subjects were 49 undergraduates, 9 of whom were excluded from the final sample for failing to meet the training criterion. The remaining 40 subjects had relatively few years of formal music lessons: 35 had music lessons for 5 years or less (mean of 1.91 years), 4 had lessons for more than 5 years (mean of 9 years), and 1 had no formal lessons but had played percussion for 12 years.

\section{Apparatus}

The subjects were tested in a double-walled sound-attenuating booth (Industrial Acoustics Co.). An ECS personal computer and custom interface controlled stimulus presentation and response recording. Two synthesizer/function generators (Hewlett-Packard $3325 \mathrm{~A}$ ) produced the sine-wave tones, which were attenuated by two attenuators (Med Associates) and turned on and off by two rise/fall switches (Med Associates). Stimuli were presented with a stereo amplifier (Marantz 1070) and loudspeaker (Avant 2AX). The experimenter initiated trials with a touch-sensitive control device connected to the computer; the same device was used to record responses. Illumination and activation of mechanical toys housed in a Plexiglas box under the loudspeaker provided feedback about correct responding.

\section{Stimuli}

Tone patterns consisted of five contiguous pure tones of equal intensity, each $400 \mathrm{msec}$ in duration with 10 -msec linear onset and offset ramps (with respect to amplitude). The average sound level was $75 \mathrm{~dB}(\mathrm{~A})$ at the approximate position of the listener's head. Each pattern had an alternating low tone and high tone; the first, third, and fifth tones were identical (the low tones), as were the 
second and fourth tones (the high tones), resulting in a rise-fallrise-fall contour. The crucial frequency ratio was the interval between high and low tones - that is, the ratio of the frequency of the high tone (in $\mathrm{Hz}$ ) to that of the low tone.

There were eight different conditions; each involved a repeatingbackground, or standard, tone pattern and a comparison pattern with a frequency ratio different from that of the background. The comparison pattern was always one semitone smaller or larger than the background pattern. Frequency ratios and interval sizes of background and comparison patterns for each condition are shown in Table 1 . Conditions 1 and 2 had a background pattern that was perfectly consonant (simple frequency ratio of $3: 2$ or $4: 3$ ) and a comparison pattern that was dissonant (complex frequency ratio of 45:32). Conditions 3 and 4 had a background pattern that was perfectly consonant $(3: 2$ or $4: 3)$ and a comparison pattern that was imperfectly consonant (moderately simple frequency ratio of $8: 5$ or $5: 4)$. Conditions 5 and 6 had a background pattern that was imperfectly consonant $(5: 4$ or $8: 5)$ and a comparison pattern that was perfectly consonant $(4: 3$ or $3: 2$ ). Conditions 7 and 8 had a background pattern that was dissonant (45:32) and a comparison pattern that was perfectly consonant $(4: 3$ or $3: 2)$. For each pair of conditions (e.g., Conditions 1 and 2), increases or decreases in interval size were counterbalanced across the frequency ratio manipulation (e.g., in Condition 1, the simple frequency ratio was larger than the complex ratio; in Condition 2, the simple ratio was smaller than the complex ratio). Table 2 provides a sample stimulus for each of the frequency ratios tested. Although the absolute size of the difference between background and comparison patterns was identical (i.e., one semitone) across all conditions, the size of the semitone change relative to the background pattern ranged from a low of $1 / 8(12.5 \%)$ in Condition 6 (background interval of 8 semitones) to a high of $1 / 4(25 \%)$ in Condition 5 (background interval of 4 semitones).

Repetitions of the background pattern were separated by 1,200 msec silent intervals and were transposed (same frequency ratio between high and low tones, different absolute frequencies) 2 semitones higher or lower in pitch between consecutive patterns. On change trials, the comparison pattern (low tone similarly transposed, high tone displaced from an exact transposition by one semitone) replaced the background for a single presentation. The first tone of the lowest possible transposition was $300 \mathrm{~Hz}$ (slightly higher than D4, or $294 \mathrm{~Hz}$ ). Other possible starting tones were 2, $4,6,8$, or 10 semitones higher. Figure 1 illustrates, in musical notation, an example of 16 possible consecutive patterns in Condition 1 (background 3:2, comparison 45:32).

Each condition began with a training phase in which the background pattern was identical to that of the test phase, but the comparison pattern had a much larger change (an octave downward) to the second and fourth tones. Thus, the comparison pattern in the training phase had a different contour (fall-rise-fall-rise) from

Table 1

Frequency Ratio and Interval Size (in Semitones) of Background and Comparison Patterns

\begin{tabular}{cccccc}
\hline & \multicolumn{4}{c}{ Pattern } \\
\cline { 2 - 3 } & \multicolumn{2}{c}{ Background } & & \multicolumn{2}{c}{ Comparison } \\
\cline { 2 - 3 } \cline { 5 - 6 } Condition & $\begin{array}{c}\text { Frequency } \\
\text { Ratio }\end{array}$ & $\begin{array}{c}\text { Interval } \\
\text { Size }\end{array}$ & & $\begin{array}{c}\text { Frequency } \\
\text { Ratio }\end{array}$ & $\begin{array}{c}\text { Interval } \\
\text { Size }\end{array}$ \\
\hline 1 & $3: 2$ & 7 & & $45: 32$ & 6 \\
2 & $4: 3$ & 5 & & $45: 32$ & 6 \\
3 & $3: 2$ & 7 & & $8: 5$ & 8 \\
4 & $4: 3$ & 5 & & $5: 4$ & 4 \\
5 & $5: 4$ & 4 & & $4: 3$ & 5 \\
6 & $8: 5$ & 8 & & $3: 2$ & 7 \\
7 & $45: 32$ & 6 & $4: 3$ & 5 \\
8 & $45: 32$ & 6 & $3: 2$ & 7 \\
\hline
\end{tabular}

Table 2

Sample Stimuli for Each of the Frequency Ratios (Lowest Possible Transposition)

\begin{tabular}{cccccc}
\hline Frequency & \multicolumn{5}{c}{ Sample Stimulus } \\
\cline { 2 - 6 } Ratio & Tone 1 & Tone 2 & Tone 3 & Tone 4 & Tone 5 \\
\hline $3: 2$ & 300 & 450 & 300 & 450 & 300 \\
$4: 3$ & 300 & 400 & 300 & 400 & 300 \\
$5: 4$ & 300 & 375 & 300 & 375 & 300 \\
$8: 5$ & 300 & 480 & 300 & 480 & 300 \\
$45: 32$ & 300 & 422 & 300 & 422 & 300 \\
\hline
\end{tabular}

Note-All tones are expressed in hertz.

that of the background, but the component tones had the same tone chroma in the same order as those of the background pattern.

\section{Procedure}

Each listener was tested individually in one condition, ${ }^{1}$ resulting in 5 listeners being tested in each of the eight conditions. We used a modified version of the go/no-go procedure typically used for infant listeners, so that the resulting adult data could provide a basis for future comparisons with infants (see Trainor \& Trehub, 1992, 1993a). Participants heard the background pattern presented repeatedly in transposition and were instructed to raise their hand when they heard a different pattern. The test phase consisted of 60 trials initiated by the experimenter when the subject was judged to be attentive and facing directly ahead: $\mathbf{3 0}$ change trials and $\mathbf{3 0}$ control (no-change) trials, with a minimum of three background patterns occurring between trials. The order of change and control trials was randomized, constrained by a maximum of two consecutive control trials. On change trials, the comparison pattern was substituted for the background pattern. Control trials consisted of another presentation of the background pattern and were, as such, identical to the background. Trials were not marked in any way to make them distinct from background patterns (except for the change trials). Although the experimenter, who was blind to the nature of trials, recorded all hand raises, only those occurring after the onset of the second (potentially changed) tone of a trial pattern and before the onset of the following pattern (a 2,800-msec response window) were scored by the computer. Feedback for correct responses (i.e., hand raise during change trials) consisted of the illumination and activation of a mechanical toy for $2,500 \mathrm{msec}$. The test phase of each condition took 13-14 min, during which approximately 250 patterns were presented (approximately $12 \%$ of these were change trials).

Prior to the test phase, the subjects participated in a training phase, which was identical to the test phase except that only change trials were included and the comparison pattern embodied a relatively larger change. To draw attention to the changes, the first two training trials were presented at an intensity $5 \mathrm{~dB}$ greater than that of the background pattern. Incorrect responding on two successive trials resulted in a $5-\mathrm{dB}$ increase on the next two trials (to a maximum 10-dB difference between test and background intensity). Correct responding on any training trial resulted in a 5-dB decrease until comparison and background patterns were at equivalent intensity. The participants were required to meet a training criterion of four consecutive correct responses (with change and background patterns at equal intensity) within 20 trials; those who failed to do so were excluded from the subsequent test phase.

\section{RESULTS}

Mean hits and false alarms for each condition are provided in Table 3. For each subject in each condition, proportions of correctly identified change trials (hits) and incorrectly identified control trials (false alarms) were 

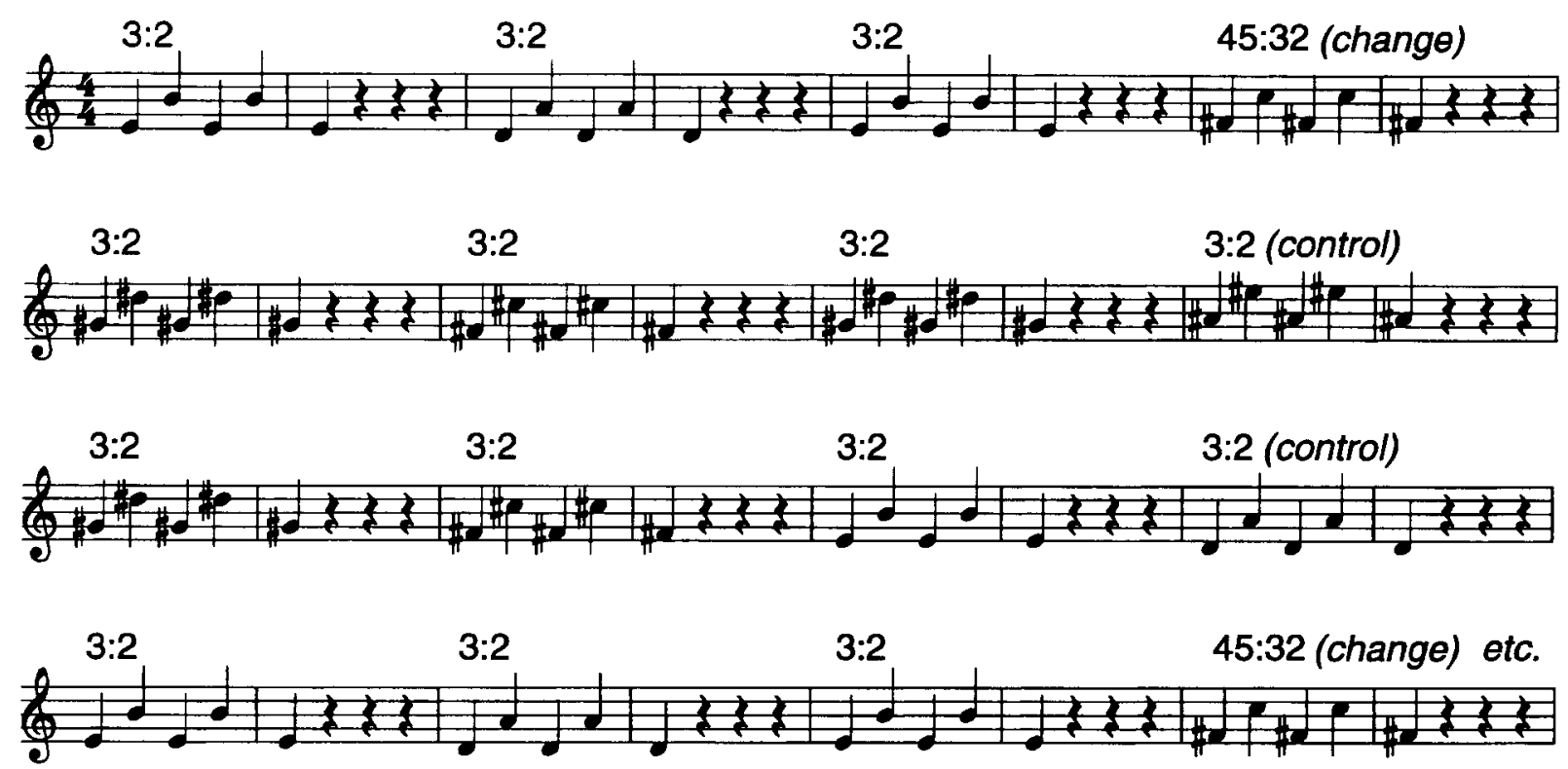

Figure 1. An example of 16 consecutive patterns from Condition 1 (background pattern with a frequency ratio of 3:2; comparison pattern with a ratio of $45: 32$ ).

transformed to $d^{\prime}$ scores according to yes/no signal detection tables (Elliott, 1964). The $d^{\prime}$ transformation eliminates listener response bias if the assumptions of normality and homogeneity of variance are reasonably valid (Green \& Swets, 1974). To counteract the problem of potentially infinite $d^{\prime}$ scores for proportions of 0 or 1 , all proportions were adjusted by adding .5 to the number of responses and dividing by the number of trials plus 1 (i.e., 31). This additional transformation has little effect on $d^{\prime}$ scores and preserves the original rank ordering (Thorpe, Trehub, Morrongiello, \& Bull, 1988).

Mean $d^{\prime}$ scores (averaged across individual listeners' scores) as a function of the eight experimental conditions are shown in Figure 2. Conditions are ordered along the abscissa (from Condition 1 to Condition 8) so that the frequency ratio of the comparison pattern increases in simplicity relative to the background pattern with increasing distance from the ordinate. (The leftmost condition, Condition 1, represents the background pattern with the simplest frequency ratio [3:2] coupled with the comparison pattern with the most complex ratio [45:32]; the rightmost condition, Condition 8, represents the background pattern with the most complex ratio [45:32] coupled with the comparison pattern with the simplest ratio [3:2].)

A preliminary analysis revealed that the absolute magnitude of the background interval, which varied from a low of 4 semitones in Condition 5 to a high of 8 semitones in Condition 6, was unrelated to performance $(r=$ $.001, p=1, N=40$ ). Thus, the size of the semitone change relative to the background pattern (ranging from $12.5 \%$ to $25 \%$ ) did not affect performance. Accordingly, relative size of change was not considered further.
A one-way analysis of variance (between-subjects) revealed performance variations among the eight experimental conditions $[F(7,32)=3.60, p<.01]$; differences between conditions were examined by means of seven planned orthogonal contrasts. The first examined performance differences between Conditions 1-4 (simpleratio background of $3: 2$ or $4: 3$; complex-ratio comparison of 45:32 or moderately simple-ratio comparison of $8: 5$ or 5:4) and Conditions 5-8 (complex-ratio background of 45:32 or moderately simple-ratio background of $8: 5$ or $5: 4$; simple-ratio comparison of $3: 2$ or $4: 3$ ). The contrast indicated that $d^{\prime}$ scores in Conditions $1-4$ were significantly higher than those in Conditions $5-8[F(1,32)$ $=6.67, p<.05]$. The second contrast, restricted to conditions in which the background pattern had a simple frequency ratio ( $3: 2$ or $4: 3$, Conditions $1-4)$, revealed that performance was superior when the comparison patterns had a complex ratio (45:32, Conditions 1 and 2 ) as opposed to a moderately simple ratio (8:5 or $5: 4$, Conditions 3 and 4$)[F(1,32)=10.08, p<.005]$. The third contrast, which concerned conditions in which the com-

Table 3

Averaged Hit Rates and False-Alarm Rates as a Function of Condition

\begin{tabular}{cccc}
\hline Condition & Ratios & Hit Rate (\%) & False-Alarm Rate (\%) \\
\hline 1 & $3: 2$ vs. $45: 32$ & 95.3 & 5.3 \\
2 & $4: 3$ vs. $45: 32$ & 87.3 & 16.0 \\
3 & $3: 2$ vs. $8: 5$ & 62.7 & 8.7 \\
4 & $4: 3$ vs. $5: 4$ & 54.7 & 12.0 \\
5 & $5: 4$ vs. $4: 3$ & 66.0 & 2.7 \\
6 & $8: 5$ vs. $3: 2$ & 52.0 & 4.0 \\
7 & $45: 32$ vs. $4: 3$ & 51.3 & 12.0 \\
8 & $45: 32$ vs. $3: 2$ & 30.0 & 12.7 \\
\hline
\end{tabular}




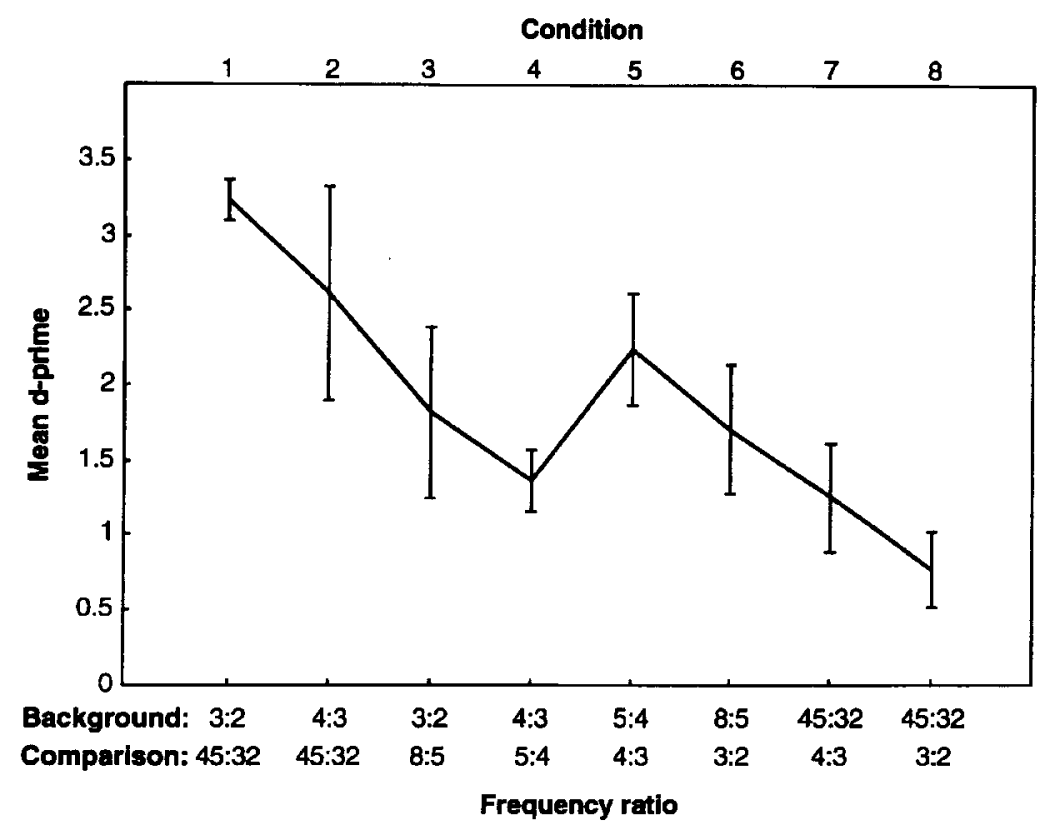

Figure 2. Mean $d^{\prime}$ scores as a function of the eight experimental conditions. Conditions are ordered along the abscissa so that the simplicity of the frequency ratio of the background pattern relative to the comparison pattern decreases with increasing distance from the ordinate. Error bars represent standard errors.

parison pattern had a simple frequency ratio ( $3: 2$ or $4: 3$, Conditions 5-8), revealed improved performance when the background patterns had a moderately simple ratio (8:5 or $5: 4$, Conditions 5 and 6$)$ rather than a complex ratio $(45: 32$, Conditions 7 and 8$)[F(1,32)=5.27, p<$ $.05]$. Thus, performance was best when the background pattern had a simple frequency ratio, intermediate when it had a moderately simple ratio, and poorest when it had a complex ratio. Conversely, performance was best when the comparison pattern had a complex ratio, intermediate when it had a moderately simple ratio, and poorest when it had a simple ratio.

The fourth contrast indicated that mean $d^{\prime}$ scores did not differ as a function of whether the comparison pattern was smaller (Conditions 1, 4, 6, and 7) or larger (Conditions 2, 3, 5, and 8) than the background pattern $[F(1,32)<1]$. The fifth, sixth, and seventh contrasts showed that the effects tested in the first, second, and third contrasts did not interact significantly with the fourth contrast $[F(1,32)<1, F(1,32)=1.65$, and $F(1,32)=$ 1.46 , respectively].

\section{DISCUSSION}

The present investigation provided unequivocal evidence of the effects of frequency ratios on the discrimination of changes to pure tone sequences. Changes from patterns with simple frequency ratios to those with complex ratios were more readily detected than were changes from complex to simple ratios. Moreover, larger effects were associated with greater differences in ratio simplicity between the background and comparison patterns.
Performance was not differentially affected by the specific increases or decreases in interval size tested in the present study or by the size of changes tested relative to the background pattern.

Effects of frequency ratios on the processing of sequential pure tones imply some contribution of acoustic factors to musical consonance, or tone compatibility. Shepard (1982) proposed that the influence of "significant" musical intervals (octaves, fifths, fourths, and thirds - intervals with simple frequency ratios) on pitch perception is found reliably only when complex tones are presented in musical contexts to musically sophisticated listeners. Our results indicate, however, that ratio simplicity can affect relatively unsophisticated listeners' perception of sequential pure tones in nonmusical contexts. Thus, our findings point to the need for redefining traditional distinctions between sensory and musical consonance to account for acoustically generated effects that are independent of overlapping critical bands.

The present results may arise, in part, from culturespecific factors, because of the fundamental role of $3: 2$, $4: 3$, and 5:4 frequency ratios in Western harmony and scale structure. Indeed, the local peak in performance in Condition 5 (5:4 background, 4:3 comparison; see Figure 2) may reflect exposure to Western harmony, in which simultaneous tones are grouped more often in ratios of 5:4 (major thirds) than 4:3 (perfect fourths). Overlearning of Western musical relations may affect the perception of tone sequences even in musically impoverished contexts by relatively unsophisticated listeners. From this perspective, some processing biases might be a consequence of specific musical structures. The principal 
limitation of this explanation is its failure to account for similarities among musical cultures, viewing the crosscultural importance of simple frequency ratios, such as 2:1, 3:2, and 4:3 (Meyer, 1956; Sachs, 1943), as mere coincidence. Future developmental and cross-cultural research could clarify the relative contributions of inherent processing predispositions and musical enculturation to the perceptual asymmetries observed in the present investigation.

Why did we find effects of the simplicity of frequency ratios that have eluded other investigators? Previous research on the perceived consonance of pure tones has focused largely on simultaneous tones (e.g., Kameoka \& Kuriyagawa, 1969; Plomp \& Levelt, 1965; van de Geer, Levelt, \& Plomp, 1962), usually requiring listeners to provide qualitative ratings (e.g., pleasantness, consonance) of stimuli. Such evaluative judgments may be less sensitive to perceptual processing predispositions and more sensitive to musical training than the simple discrimination task of the present investigation. Moreover, the use of intervals varying greatly in size (e.g., Kallman, 1982; Levelt, van de Geer, \& Plomp, 1966) may have enhanced size distinctions at the expense of intervallic quality, whereas our uniform interval size changes (one semitone) may have highlighted intervallic quality. Perhaps the evaluative judgments of other studies engaged explicit processing, or declarative knowledge, in contrast to the simple discrimination task of the present investigation, which engaged implicit processing, or procedural knowledge (see also Dowling, 1993). Burns and Ward's (1978, Experiment 4) divergent findings could, therefore, be attributable to the difficulty of their task. For example, their stimulus patterns were sequences consisting of only two tones, and their listeners were asked to identify which of two intervals was wider (not whether they differed). Moreover, differences in interval size were less than a semitone, and transpositions between intervals were not in multiples of semitones. Thus, despite the inherent conservatism of a test of consonance with sequential tones, our simple discrimination task may be considerably more sensitive to perceptual processing nuances than are the rating, judgment, and identification tasks of previous investigations.

Our finding of perceptual asymmetries, although similar in some respects to previous reports, is unique in that it relates specifically to intervals (i.e., two tones) in musically impoverished contexts. Other researchers have found asymmetries in similarity ratings of musical tones (Krumhansl, 1979), chord sequences (Bharucha \& Krumhansl, 1983), and pairs of melodies (Bartlett \& Dowling, 1988). Moreover, rhythmic tone sequences are differentiated more readily from sequences with irregular rhythms when the rhythmic sequences are presented first (Bharucha \& Pryor, 1986).

In the present research, simple frequency ratios may have fostered a relatively stable representation of the background intervals, making the changes more noticeable. By contrast, background intervals with complex ratios may have been anchored to, or resolved by, the subsequent simple ratio intervals, much like the anchoring of unstable to stable tones in melodies (Bharucha, 1984). Anchoring effects such as these might obscure the interval changes. An alternative way of conceptualizing the issue is to view complex ratios as distinct or salient, especially in the context of simple ratios. Indeed, if the simplicity of frequency ratios is an acoustic determinant of tone compatibility, then the dissonance of incompatible tones may make them particularly salient.

Our finding of perceptual asymmetries is also consistent with the notion of musical prototypes (Trehub \& Unyk, 1991). In music, intervals with small integer frequency ratios may be prototypical (Jones, 1990) and used as "cognitive reference points" (Rosch, 1975), perhaps independently of learning and exposure. Conversely, intervals with complex frequency ratios, such as 45:32, may be nonprototypical. Listeners in the present study may have been unable to form a stable representation of the $45: 32$ ratio, considering it, perhaps, as a poor example of a more familiar interval (i.e., either $3: 2$ or $4: 3$ ). The possibility that acoustic properties underlie prototypical musical intervals warrants further investigation.

\section{REFERENCES}

AldWell, E., \& Schachter, C. (1989). Harmony and voice leading (2nd ed.). San Diego: Harcourt Brace Jovanovich.

ALLEN, D. (1967). Octave discriminability of musical and non-musical subjects. Psychonomic Science, 7, 421-422.

AttNeave, F., \& Olson, R. K. (1971). Pitch as a medium: A new approach to psychophysical scaling. American Journal of Psychology, 84, 147-166.

Bartlett, J. C., \& Dowling, W. J. (1988). Scale structure and similarity of melodies. Music Perception, 5, 285-314.

BHARUCHA, J. J. (1984). Anchoring effects in music: The resolution of dissonance. Cognitive Psychology, 16, 485-518.

Bharucha, J. J., \& KRUMhansL, C. L. (1983). The representation of harmonic structure in music: Hierarchies of stability as a function of context. Cognition, 13, 63-102.

Bharucha, J. J., \& PryoR, J. H. (1986). Disrupting the isochrony underlying rhythm: An asymmetry in discrimination. Perception \& Psychophysics, 40, 137-141.

Bregman, A. S. (1990). Auditory scene analysis. Cambridge, MA: MIT Press.

Burns, E. M., \& WARD, W. D. (1978). Categorical perceptionPhenomenon or epiphenomenon: Evidence from experiments in the perception of melodic musical intervals. Journal of the Acoustical Society of America, 63, 456-468.

Cohen, A. J., ThorPe, L. A., \& TRehub, S. E. (1987). Infants' perception of musical relations in short transposed tone sequences. Canadian Journal of Psychology, 41, 33-47.

Demany, L., \& Armand, F. (1984). The perceptual reality of tone chroma in early infancy. Journal of the Acoustical Society of America, 76, 57-66.

DivenYI, P. L., \& HiRsh, I. J. (1974). Identification of temporal order in three-tone sequences. Journal of the Acoustical Society of America, 56, 144-151.

Dowling, W. J. (1993). Procedural and declarative knowledge in music cognition and education. In T. J. Tighe \& W. J. Dowling (Eds.), Psychology and music: The understanding of melody and rhythm (pp. 5-18). Hillsdale, NJ: Erlbaum.

Dowling, W. J., \& Harwood, D. L. (1986). Music cognition. San Diego: Academic Press 
Elliott, P. B. (1964). Tables of $d^{\prime}$. In J. A. Swets (Ed.), Signal detection and recognition by human observers: Contemporary readings (Appendix 1, pp. 651-684). New York: Wiley.

GreEN, D. M., \& SwETs, J. A. (1974). Signal detection theory and psychophysics. New York: Wiley.

Houtsma, A. J. M. (1968). Discrimination of frequency ratios. Journal of the Acoustical Society of America, 44, S383.

JONES, M. R. (1990). Learning and the development of expectancies: An interactionist approach. Psychomusicology, 9, 193-228.

KALLMAN, H. J. (1982). Octave equivalence as measured by similarity ratings. Perception \& Psychophysics, 32, 37-49.

Kameoka, A., \& KurIYagaWA, M. (1969). Consonance theory part I: Consonance of dyads. Journal of the Acoustical Society of America, 45, 1451-1459.

KRUMHANSL, C. L. (1979). The psychological representation of musical pitch in a tonal context. Cognitive Psychology, 11, 346-374.

LERDAHL, F., \& JACKENDOFF, R. (1983). A generative theory of tonal music. Cambridge, MA: MIT Press.

LeVelt, W. J. M., vaN DE GeER, J. P., \& Plomp, R. (1966). Triadic comparisons of musical intervals. British Journal of Mathematical \& Statistical Psychology, 19, 163-179.

Macmillan, N. A., \& Creelman, C. D. (1991). Detection theory: A user's guide. New York: Cambridge University Press.

MEYER, L. B. (1956). Emotion and meaning in music. Chicago: University of Chicago Press.

Plomp, R., \& LeVELT, W. J. M. (1965). Tonal consonance and critical bandwidth. Journal of the Acoustical Society of America, 38, 548560.

Rosch, E. (1975). Cognitive reference points. Cognitive Psychology, 7, 532-547.

SACHS, C. (1943). The rise of music in the ancient world: East and West. New York: Norton.

Schellenberg, E. G., \& Trehub, S. E. (1994). Frequency ratios and the perception of tone patterns. Psychonomic Bulletin \& Review, 1, 191-201.

SHEPARD, R. N. (1982). Structural representations of musical pitch. In D. Deutsch (Ed.), The psychology of music (pp. 343-390). New York: Academic Press.

SIEGEL, J. A., \& SIEGEL, W. (1977a). Absolute identification of notes and intervals by musicians. Perception \& Psychophysics, 21, 143152.
Siegel, J. A., \& Siegel, W. (1977b). Categorical perception of tonal intervals: Musicians can't tell sharp from flat. Perception \& Psychophysics, 21, 399-407.

Thorpe, L. A., Trehub, S. E., Morrongiello, B. A., \& Bull, D. (1988). Perceptual grouping by infants and preschool children. Developmental Psychology, 24, 484-491.

ThuRLOW, W. R., \& ERCHUL, W. P. (1977). Judged similarity in pitch of octave multiples. Perception \& Psychophysics, 22, 177-182.

Trainor, L. J., \& Trehub, S. E. (1992). A comparison of infants' and adults' sensitivity to Western musical structure. Journal of Experimental Psychology: Human Perception \& Performance, 18, 394 402.

Trainor, L. J., \& Trehub, S. E. (1993a). Musical context effects in infants and adults: Key distance. Journal of Experimental Psychology: Human Perception \& Performance, 19, 615-626.

TrainoR, L. J., \& TrehUB, S. E. (1993b). What mediates infants' and adults' superior processing of the major over the augmented triad? Music Perception, 11, 185-196.

Trehub, S. E., ThORPE, L. A., \& Trainor, L. J. (1990). Infants' perception of good and bad melodies. Psychomusicology, 9, 5-15.

Trenub, S. E., \& Trainor, L. J. (1993). Listening strategies in infancy: The roots of music and language development. In S. McAdams \& E. Bigand (Eds.), Thinking in sound: The cognitive psychology of human audition (pp. 278-327). Oxford: Oxford University Press.

TREHUB, S. E., \& UNYK, A. M. (1991). Music prototypes in developmental perspective. Psychomusicology, 10, 31-45.

VAN de Geer, J. P., Levelt, W. J. M., \& Plomp, R. (1962). The connotation of musical consonance. Acta Psychologica, 20, 308-319.

\section{NOTE}

1. Listeners were originally tested in two conditions; the background and comparison patterns of the first condition were reversed for the second condition (e.g., listeners initially tested in Condition 1 were subsequently tested in Condition 8). Performance was significantly poorer in the second condition tested, either because of fatigue or because of the direct reversal of decision rules. Accordingly, all data from the second condition were excluded from further analysis.

(Manuscript received June 25, 1993; revision accepted for publication May $17,1994$. 